\title{
Impulsive Stabilization for a Class of Neural Networks with Both Time-Varying and Distributed Delays
}

\author{
Lizi Yin ${ }^{1}$ and Xiaodi $\mathbf{L i}^{2}$ \\ ${ }^{1}$ School of Science, University of Jinan, Jinan 250022, China \\ ${ }^{2}$ Department of Mathematics, Xiamen University, Xiamen 361005, China \\ Correspondence should be addressed to Lizi Yin, ss_yinlz@ujn.edu.cn \\ Received 16 January 2009; Accepted 4 March 2009 \\ Recommended by Paul Eloe
}

The impulsive control method is developed to stabilize a class of neural networks with both timevarying and distributed delays. Some exponential stability criteria are obtained by using Lyapunov functionals, stability theory, and control by impulses. A numerical example is also provided to show the effectiveness and feasibility of the impulsive control method.

Copyright (C) 2009 L. Yin and X. Li. This is an open access article distributed under the Creative Commons Attribution License, which permits unrestricted use, distribution, and reproduction in any medium, provided the original work is properly cited.

\section{Introduction}

During the last decades, neural networks such as Hopfield neural networks, cellular neural networks, Cohen-Grossberg neural networks, and bidirectional associative memory neural networks have been extensively studied. There have appeared a number of important results; see [1-13] and references therein. It is well known that the properties of stability and convergence are important in design and application of neural networks, for example, when designing a neural network to solve linear programming problems and pattern recognition problems, we foremost guarantee that the models of neural network are stable. However, it may become unstable or even divergent because the model of a system is highly uncertain or the nature of the problem itself. So it is necessary to investigate stability and convergence of neural networks from the control point of view. It is known that impulses can make unstable systems stable or, otherwise, stable systems can become unstable after impulse effects; see [14-18]. The problem of stabilizing the solutions by imposing proper impulse controls has been used in many fields such as neural network, engineering, pharmacokinetics, biotechnology, and population dynamics [19-25]. Recently, several good impulsive control 
approaches for real world systems have been proposed; see [22-32]. In [26], Yang and $\mathrm{Xu}$ investigate the global exponential stability of Cohen-Grossberg neural networks with variable delays by establishing some impulsive differential inequalities. The criteria not only present an approach to stabilize the unstable neural networks by utilizing impulsive effects but also show that the stability still remains under certain impulsive perturbations for some continuous stable neural networks. In [27], Li et al. consider the impulsive control of Lotka-Volterra predator-prey system by employing the method of Lyapunov functions. In [28], Wang and Liu investigate the impulsive stabilization of delay differential systems via the Lyapunov-Razumikhin method. However, there are few results considering the impulsive stabilization of neural networks with both time-varying and distributed delays, which is very important in theories and applications and also is a very challenging problem.

Motivated by the above discussion, in this paper, we will investigate the impulsive stabilization for a class of neural networks with both time-varying and distributed delays. Some exponential stability criteria are obtained by using Lyapunov functionals, stability theory, and control by impulses. The organization of this paper is as follows. In the next section, the problems investigated in this paper are formulated, and some preliminaries are presented. We state and prove our main results in Section 3. Then, an illustrative example is given to show the effectiveness of the obtained impulsive control method in Section 4. Finally, concluding remarks are made in Section 5.

\section{Model Description and Preliminaries}

Let $\mathbb{R}$ denote the set of real numbers, $\mathbb{R}^{n}$ the $n$-dimensional real space equipped with the Euclidean norm $|\cdot|$, and $\mathbb{Z}_{+}$the set of positive integral numbers.

Considering the following neural networks with both time-varying and distributed delays:

$$
\dot{x}_{i}(t)=-d_{i} x_{i}(t)+\sum_{j=1}^{n} a_{i j} f_{j}\left(x_{j}\left(t-\tau_{j}(t)\right)\right)+\sum_{j=1}^{n} b_{i j} g_{j}\left(\int_{0}^{\omega} K_{i j}(s) x_{j}(t-s) d s\right)+I_{i}, \quad t \geq t_{0}, i \in \Lambda,
$$

where $\Lambda=\{1,2, \ldots, n\}, n \geq 2$ corresponds to the number of units in a neural network, $x_{i}$ is the state variable of the $i$ th neuron, $d_{i}>0$ denotes the passive delay rates, $a_{i j}, b_{i j}$ denote the connection weights of the unit $j$ on the unit $i, f_{j}, g_{j}$ are the activation functions of the neurons, $I_{i}$ is the input of the unit $i$, and $\tau_{j}(t)$ is the transmission delay of the $j$ th neuron such that $0 \leq \tau_{j}(t) \leq \tau, \dot{\tau}_{j}(t) \leq \rho<1, j \in \Lambda, t \geq t_{0}$, where $\tau, \rho$ and $\omega$ are some constants. And the system (2.1) is supplemented with initial values given by the form

$$
x_{i}\left(t_{0}+\theta\right)=\phi_{i}(\theta), \quad-\max \{\tau, \omega\} \leq \theta \leq 0,
$$

where $\phi_{i} \in \mathbb{C}, \mathbb{C}$ denotes piecewise continuous functions defined on $[-\max \{\tau, \omega\}, 0]$. For $x \in \mathbb{R}^{n}, \phi \in \mathbb{C}^{n}$, let $\|u\|=\sum_{i=1}^{n}\left|u_{i}\right|,\|\phi\|=\sup _{-\max \{\tau, \omega\} \leq s \leq 0}\left(\sum_{i=1}^{n}\left|\phi_{i}\right|\right)$. 
We also consider the impulses at times $t_{k}, k \in \mathbb{Z}_{+}$,

$$
\Delta x_{i}\left(t_{k}\right)=x_{i}\left(t_{k}\right)-x_{i}\left(t_{k}^{-}\right)=\gamma_{i k} x_{i}\left(t_{k}^{-}\right), \quad i \in \Lambda,
$$

where $\gamma_{i k} \geq-1$ are some undetermined constants.

Throughout this paper, we assume the following.

$\left(H_{1}\right) f_{j}, g_{j}$ are bounded and satisfy the following property:

$$
\left|f_{j}\left(s_{1}\right)-f_{j}\left(s_{2}\right)\right| \leq L_{j}^{f}\left|s_{1}-s_{2}\right|, \quad\left|g_{j}\left(s_{1}\right)-g_{j}\left(s_{2}\right)\right| \leq L_{j}^{g}\left|s_{1}-s_{2}\right|, \quad \forall s_{1}, s_{2} \in \mathbb{R}, j \in \Lambda,
$$

where $L_{j}^{f}, L_{j}^{g}$ are constants for $j \in \Lambda$.

$\left(H_{2}\right)$ The delay kernels $K_{i j}:[0, \omega) \rightarrow \mathbb{R}_{+}, i, j \in \Lambda$, are piecewise continuous and satisfy $K_{i j}(s) \leq \mathcal{K}(s)$ for all $i, j \in \Lambda, s \in[0, \omega)$, where $\mathcal{K}(s):[0, \omega) \rightarrow \mathbb{R}_{+}$is continuous and integrable.

$\left(H_{3}\right)$ The impulse times $t_{k}$ satisfy $0 \leq t_{0}<t_{1}<\cdots<t_{k}<\cdots, \lim _{k \rightarrow+\infty} t_{k}=+\infty$.

Since $\left(H_{1}\right)$ and $\left(H_{2}\right)$ hold, by employing the well-known Brouwer's fixed point theorem, one can easily prove that there exists a unique equilibrium point for system (2.1).

Assume that $x^{\star}$ is an equilibrium solution of system (2.1), then the transformation $u_{i}=x_{i}-x_{i}^{\star}, i \in \Lambda$ puts system (2.1) and (2.2) into the following form:

$$
\begin{aligned}
\dot{u}_{i}(t)= & -d_{i} u_{i}(t)+\sum_{j=1}^{n} a_{i j} f_{j}^{\star}\left(u_{j}\left(t-\tau_{j}(t)\right)\right) \\
& +\sum_{j=1}^{n} b_{i j} g_{j}^{\star}\left(\int_{0}^{\omega} K_{i j}(s) u_{j}(t-s) d s\right), \quad t \geq t_{0}, \\
u_{i}\left(t_{0}+\theta\right)= & \varphi_{i}(\theta), \quad-\max \{\tau, \omega\} \leq \theta \leq 0, \quad i \in \Lambda,
\end{aligned}
$$

where $f_{j}^{\star}\left(u_{j}\right)=f_{j}\left(u_{j}+x_{j}^{\star}\right)-f_{j}\left(x_{j}^{\star}\right), g_{j}^{\star}\left(u_{j}\right)=g_{j}\left(u_{j}+x_{j}^{\star}\right)-g_{j}\left(x_{j}^{\star}\right), \varphi_{i}(s)=\phi_{i}(s)-x_{i}^{\star}$.

\section{Impulsive Stabilization of the Equilibrium Solution}

Theorem 3.1. Assume that $\left(H_{1}\right)-\left(H_{3}\right)$ hold, then the equilibrium point of the system (2.1) can be exponentially stabilized by impulses if one of the following conditions hold.

$$
\left(H_{4}\right) \mathbb{A}<0 .
$$


$\left(H_{5}\right) \mathbb{A} \geq 0$ and $\exp [\mathbb{A} \max \{\tau, \omega\}] \cdot \mathbb{B}<1$, where

$$
\begin{aligned}
& \mathbb{A}=-\min _{i \in \Lambda} d_{i}+\frac{1}{1-\rho} \sum_{i=1}^{n} \max _{j \in \Lambda}\left|a_{i j}\right| L_{j}^{f}+\sum_{i=1}^{n} \max _{j \in \Lambda}\left|b_{i j}\right| L_{j}^{g} \int_{0}^{\omega} \mathcal{K}(s) d s, \\
& \mathbb{B}=\frac{\tau}{1-\rho} \sum_{i=1}^{n} \max _{j \in \Lambda}\left|a_{i j}\right| L_{j}^{f}+\sum_{i=1}^{n} \max _{j \in \Lambda}\left|b_{i j}\right| L_{j}^{g} \int_{0}^{\omega} \mathcal{K}(s) s d s .
\end{aligned}
$$

Proof. First, we consider the following positive definite Lyapunov functional:

$$
\begin{aligned}
V(t)= & \sum_{i=1}^{n}\left|u_{i}(t)\right|+\frac{1}{1-\rho} \sum_{i=1}^{n} \sum_{j=1}^{n}\left|a_{i j}\right| \int_{t-\tau_{j}(t)}^{t}\left|f_{j}^{\star}\left(u_{j}(s)\right)\right| d s \\
& +\sum_{i=1}^{n} \sum_{j=1}^{n}\left|b_{i j}\right| L_{j}^{g} \int_{0}^{\omega} K_{i j}(s) \int_{t-s}^{t}\left|u_{j}(v)\right| d v d s .
\end{aligned}
$$

Then we can compute that

$$
\begin{aligned}
& \sum_{i=1}^{n}\left|u_{i}(t)\right| \leq V(t) \\
& \leq \sum_{i=1}^{n}\left|u_{i}(t)\right|+\frac{1}{1-\rho} \sum_{i=1}^{n} \sum_{j=1}^{n}\left|a_{i j}\right| L_{j}^{f} \int_{t-\tau_{j}(t)}^{t}\left|u_{j}(s)\right| d s \\
&+\sum_{i=1}^{n} \sum_{j=1}^{n}\left|b_{i j}\right| L_{j}^{g} \int_{0}^{\omega} \mathcal{K}(s) \int_{t-s}^{t}\left|u_{j}(v)\right| d v d s \\
& \leq \sum_{i=1}^{n}\left|u_{i}(t)\right|+\frac{1}{1-\rho} \sum_{i=1}^{n}\left(\max _{j \in \Lambda}\left|a_{i j}\right| L_{j}^{f}\right) \sum_{j=1}^{n} \int_{t-\tau_{j}(t)}^{t}\left|u_{j}(s)\right| d s \\
&+\sum_{i=1}^{n}\left(\max _{j \in \Lambda}\left|b_{i j}\right| L_{j}^{g}\right) \int_{0}^{\omega} \mathcal{K}(s) \int_{t-s}^{t} \sum_{j=1}^{n}\left|u_{j}(v)\right| d v d s \\
& \leq \sum_{i=1}^{n}\left|u_{i}(t)\right|+\frac{1}{1-\rho} \sum_{i=1}^{n}\left(\max _{j \in \Lambda}\left|a_{i j}\right| L_{j}^{f}\right) \int_{t-\tau}^{t} \sum_{j=1}^{n}\left|u_{j}(s)\right| d s \\
&+\sum_{i=1}^{n}\left(\max _{j \in \Lambda}\left|b_{i j}\right| L_{j}^{g}\right) \int_{0}^{\omega} \mathcal{K}(s) s d s \sup _{t-\omega \leq v \leq t}\left(\sum_{j=1}^{n}\left|u_{j}(v)\right|\right) \\
& \leq\left\{1+\frac{\tau}{1-\rho} \sum_{i=1}^{n}\left(\max _{j \in \Lambda}\left|a_{i j}\right| L_{j}^{f}\right)+\sum_{i=1}^{n} \max _{j \in \Lambda}\left(\left|b_{i j}\right| L_{j}^{g}\right) \int_{0}^{\omega} \mathcal{K}(s) s d s\right\} \\
& \times \sup _{t-\max \{\tau, \omega\} \leq s \leq t}\left(\sum_{i=1}^{n}\left|u_{i}(s)\right|\right) \\
& \sup _{t-\max \{\tau, \omega\} \leq s \leq t}\left(\sum_{i=1}^{n}\left|u_{i}(s)\right|\right), t_{t} .
\end{aligned}
$$


The time derivative of $V$ along the trajectories of system (2.5) is obtained as

$$
\begin{aligned}
& D^{+} V(t) \leq-\sum_{i=1}^{n} d_{i}\left|u_{i}(t)\right|+\sum_{i=1}^{n} \sum_{j=1}^{n}\left|a_{i j}\right|\left|f_{j}^{\star}\left(u_{j}\left(t-\tau_{j}(t)\right)\right)\right| \\
& +\sum_{i=1}^{n} \sum_{j=1}^{n}\left|b_{i j}\right|\left|g_{j}^{\star}\left(\int_{0}^{\omega} K_{i j}(s) u_{j}(t-s) d s\right)\right| \\
& +\frac{1}{1-\rho} \sum_{i=1}^{n} \sum_{j=1}^{n}\left|a_{i j}\right|\left[\left|f_{j}^{\star}\left(u_{j}(t)\right)\right|-\left|f_{j}^{\star}\left(u_{j}\left(t-\tau_{j}(t)\right)\right)\right|\left(1-\dot{\tau}_{j}(t)\right)\right] \\
& +\sum_{i=1}^{n} \sum_{j=1}^{n}\left|b_{i j}\right| L_{j}^{g} \int_{0}^{\omega} K_{i j}(s)\left[\left|u_{j}(t)\right|-\left|u_{j}(t-s)\right|\right] d s \\
& \leq-\sum_{i=1}^{n} d_{i}\left|u_{i}(t)\right|+\sum_{i=1}^{n} \sum_{j=1}^{n}\left|a_{i j}\right|\left|f_{j}^{\star}\left(u_{j}\left(t-\tau_{j}(t)\right)\right)\right|+\sum_{i=1}^{n} \sum_{j=1}^{n}\left|b_{i j}\right| L_{j}^{g} \int_{0}^{\omega} K_{i j}(s)\left|u_{j}(t-s)\right| d s \\
& +\frac{1}{1-\rho} \sum_{i=1}^{n} \sum_{j=1}^{n}\left|a_{i j}\right|\left|f_{j}^{\star}\left(u_{j}(t)\right)\right|-\frac{1-\dot{\tau}_{j}(t)}{1-\rho} \sum_{i=1}^{n} \sum_{j=1}^{n}\left|a_{i j}\right|\left|f_{j}^{\star}\left(u_{j}\left(t-\tau_{j}(t)\right)\right)\right| \\
& +\sum_{i=1}^{n} \sum_{j=1}^{n}\left|b_{i j}\right| L_{j}^{g} \int_{0}^{\omega} K_{i j}(s)\left[\left|u_{j}(t)\right|-\left|u_{j}(t-s)\right|\right] d s \\
& \leq-\sum_{i=1}^{n} d_{i}\left|u_{i}(t)\right|+\frac{1}{1-\rho} \sum_{i=1}^{n} \sum_{j=1}^{n}\left|a_{i j}\right|\left|f_{j}^{\star}\left(u_{j}(t)\right)\right|+\sum_{i=1}^{n} \sum_{j=1}^{n}\left|b_{i j}\right| L_{j}^{g} \int_{0}^{\omega} K_{i j}(s)\left|u_{j}(t)\right| d s \\
& \leq-\min _{i \in \Lambda} d_{i} \sum_{i=1}^{n}\left|u_{i}(t)\right|+\frac{1}{1-\rho} \sum_{i=1}^{n} \sum_{j=1}^{n}\left|a_{i j}\right| L_{j}^{f}\left|u_{j}(t)\right|+\sum_{i=1}^{n} \sum_{j=1}^{n}\left|b_{i j}\right| L_{j}^{g}\left|u_{j}(t)\right| \int_{0}^{\omega} \mathcal{K}(s) d s \\
& \leq\left\{-\min _{i \in \Lambda} d_{i}+\frac{1}{1-\rho} \sum_{i=1}^{n} \max _{i \in \Lambda}\left|a_{i j}\right| L_{j}^{f}+\sum_{i=1}^{n} \max _{i \in \Lambda}\left|b_{i j}\right| L_{j}^{g} \int_{0}^{\omega} \mathcal{K}(s) d s\right\} \sum_{i=1}^{n}\left|u_{i}(t)\right| \\
& \leq \mathbb{A} V(t), \quad t \geq t_{0} .
\end{aligned}
$$

Next we will consider conditions $\left(H_{4}\right)$ and $\left(H_{5}\right)$, respectively.

Case 1. If $\left(H_{4}\right)$ holds, that is, $\mathbb{A}<0$, then by (3.3) and (3.4), we get

$$
\sum_{i=1}^{n}\left|u_{i}(t)\right| \leq V(t) \leq V\left(t_{0}\right) \exp \left[\mathbb{A}\left(t-t_{0}\right)\right], \quad t \geq t_{0},
$$

which implies that the equilibrium point of the system (2.1) is exponentially stable without impulses. So the conclusion of Theorem 3.1 holds obviously. 
Case 2. If $\left(H_{5}\right)$ holds, then there exist $\varepsilon^{\star}>0$ and $\eta \geq \max \{\tau, \omega\}$ such that

$$
\mathbb{B} \leq \exp \left[-\varepsilon^{\star}(\eta+\max \{\tau, \omega\})\right] \exp [-\mathbb{A} \eta]
$$

Then one may choose a sequence $\left\{t_{k}\right\}_{k \in \mathbb{Z}_{+}}$such that $\max \{\tau, \omega\} \leq t_{k}-t_{k-1} \leq \eta$ and define

$$
\gamma_{i k}=\exp \left[-\varepsilon^{\star}\left(t_{k+1}-t_{k}+\max \{\tau, \omega\}\right)\right] \cdot \exp \left[-\mathbb{A}\left(t_{k+1}-t_{k}\right)\right]-\mathbb{B}-1 \doteq \gamma_{k}
$$

It is obvious that $\gamma_{k} \geq-1$ since (3.6) holds.

For any $\varepsilon \in(0,1)$, let

$$
\delta=\min \left\{\varepsilon, \frac{\varepsilon}{\mathbb{B}+1} \exp \left[-\left(\varepsilon^{\star}+\mathbb{A}\right)\left(t_{1}-t_{0}\right)\right]\right\}
$$

For any $t_{0} \geq 0$, we can prove that for each solution $u(t)=u\left(t, t_{0}, \varphi\right)$ of system (2.5) through $\left(t_{0}, \varphi\right),\|\varphi\| \leq \delta$ implies that

$$
\sum_{i=1}^{n}\left|u_{i}(t)\right| \leq \varepsilon \exp \left[-\varepsilon^{\star}\left(t-t_{0}\right)\right], \quad t \geq t_{0} .
$$

First, for $t \in\left[t_{0}, t_{1}\right)$, by (3.4), we have

$$
V(t) \leq V\left(t_{0}\right) \exp \left[\mathbb{A}\left(t-t_{0}\right)\right]
$$

Then considering (3.3) and the choice of $\delta$, we get

$$
\begin{aligned}
\sum_{i=1}^{n}\left|u_{i}(t)\right| & \leq V(t) \\
& \leq V\left(t_{0}\right) \exp \left[\mathbb{A}\left(t-t_{0}\right)\right] \\
& \leq V\left(t_{0}\right) \exp \left[\mathbb{A}\left(t_{1}-t_{0}\right)\right] \\
& \leq(1+\mathbb{B})\|\varphi\| \exp \left[\mathbb{A}\left(t_{1}-t_{0}\right)\right] \\
& \leq(1+\mathbb{B}) \delta \exp \left[\mathbb{A}\left(t_{1}-t_{0}\right)\right] \\
& \leq \varepsilon \exp \left[-\varepsilon^{\star}\left(t_{1}-t_{0}\right)\right] \\
& \leq \varepsilon \exp \left[-\varepsilon^{\star}\left(t-t_{0}\right)\right], \quad t \in\left[t_{0}, t_{1}\right)
\end{aligned}
$$

So we obtain

$$
\sum_{i=1}^{n}\left|u_{i}(t)\right| \leq \varepsilon \exp \left[-\varepsilon^{\star}\left(t-t_{0}\right)\right], \quad t \in\left[t_{0}, t_{1}\right)
$$


By the fact that $\max \{\tau, \omega\} \leq t_{k}-t_{k-1}$, we get

$$
\begin{aligned}
V\left(t_{1}\right)= & \left\{\sum_{i=1}^{n}\left|u_{i}\left(t_{1}\right)\right|+\frac{1}{1-\rho} \sum_{i=1}^{n} \sum_{j=1}^{n}\left|a_{i j}\right| \int_{t_{1}-\tau_{j}\left(t_{1}\right)}^{t_{1}}\left|f_{j}^{\star}\left(u_{j}(s)\right)\right| d s\right. \\
& \left.+\sum_{i=1}^{n} \sum_{j=1}^{n}\left|b_{i j}\right| L_{j}^{g} \int_{0}^{\omega} K_{i j}(s) \int_{t_{1}-s}^{t_{1}}\left|u_{j}(v)\right| d v d s\right\} \\
\leq & \left\{\sum_{i=1}^{n}\left|u_{i}\left(t_{1}^{-}\right)\right|\left|1+\gamma_{i 1}\right|+\frac{1}{1-\rho} \sum_{i=1}^{n} \max _{j \in \Lambda}\left|a_{i j} L_{j}^{f}\right| \int_{t_{1}-\tau}^{t_{1}} \sum_{j=1}^{n}\left|u_{j}(s)\right| d s\right. \\
& \left.+\sum_{i=1}^{n} \max _{j \in \Lambda}\left|b_{i j}\right| L_{j}^{g} \int_{0}^{\omega} K_{i j}(s) \int_{t_{1}-s}^{t_{1}} \sum_{j=1}^{n}\left|u_{j}(v)\right| d v d s\right\} \\
\leq & \left\{1+\gamma_{i 1}+\frac{\tau}{1-\rho} \sum_{i=1}^{n}\left(\max _{j \in \Lambda}\left|a_{i j}\right| L_{j}^{f}\right)\right. \\
& \left.+\sum_{i=1}^{n} \max _{j \in \Lambda}\left(\left|b_{i j}\right| L_{j}^{g}\right) \int_{0}^{\omega} \mathcal{K}(s) s d s\right\}_{t_{1}-\max \{\tau, \omega\} \leq s \leq t_{1}}\left(\sum_{i=1}^{n}\left|u_{i}(s)\right|\right) \\
\leq & \left(1+\gamma_{i 1}+\mathbb{B}\right) \sup \sup _{t_{1}-\max \{\tau, \omega\} \leq s \leq t_{1}}\left(\sum_{i=1}^{n}\left|u_{i}(s)\right|\right) \\
\leq & \left(1+\gamma_{i 1}+\mathbb{B}\right) \varepsilon \exp \left[-\varepsilon^{\star}\left(t_{1}-\max \{\tau, \omega\}-t_{0}\right)\right],
\end{aligned}
$$

which, together with (3.6) and (3.7), yields

$$
\begin{aligned}
\sum_{i=1}^{n}\left|u_{i}(t)\right| & \leq V(t) \\
& \leq V\left(t_{1}\right) \exp \left[\mathbb{A}\left(t-t_{1}\right)\right] \\
& \leq V\left(t_{1}\right) \exp \left[\mathbb{A}\left(t_{2}-t_{1}\right)\right] \\
& \leq\left(1+\gamma_{i 1}+\mathbb{B}\right) \varepsilon \exp \left[-\varepsilon^{\star}\left(t_{1}-\max \{\tau, \omega\}-t_{0}\right)\right] \exp \left[\mathbb{A}\left(t_{2}-t_{1}\right)\right] \\
& \leq \varepsilon \exp \left[-\varepsilon^{\star}\left(t_{2}-t_{0}\right)\right] \\
& \leq \varepsilon \exp \left[-\varepsilon^{\star}\left(t-t_{0}\right)\right], \quad t \in\left[t_{1}, t_{2}\right),
\end{aligned}
$$

that is,

$$
\sum_{i=1}^{n}\left|u_{i}(t)\right| \leq \varepsilon \exp \left[-\varepsilon^{\star}\left(t-t_{0}\right)\right], \quad t \in\left[t_{1}, t_{2}\right) .
$$


By following the similar inductive arguments as before, we derive that

$$
\sum_{i=1}^{n}\left|u_{i}(t)\right| \leq \varepsilon \exp \left[-\varepsilon^{\star}\left(t-t_{0}\right)\right], \quad t \geq t_{0}
$$

This completes our proof of Case 2.

The proof of Theorem 3.1 is complete.

Corollary 3.2. Assume that $\left(H_{1}\right),\left(H_{2}\right)$ hold, then the equilibrium point of system (2.1) is exponentially stable if the following condition holds:

$$
-\min _{i \in \Lambda} d_{i}+\frac{1}{1-\rho} \sum_{i=1}^{n} \max _{j \in \Lambda}\left|a_{i j}\right| L_{j}^{f}+\sum_{i=1}^{n} \max _{j \in \Lambda}\left|b_{i j}\right| L_{j}^{g} \int_{0}^{\omega} \mathcal{K}(s) d s<0
$$

Corollary 3.3. Assume that conditions in Theorem 3.1 hold, then the equilibrium point of the system (2.1) can be exponentially stabilized by periodic impulses.

Proof. In fact, we need only to choose the sequence $\left\{t_{k}\right\}_{k \in \mathbb{Z}_{+}}$such that $t_{k}-t_{k-1}=\eta \geq \max \{\tau, \omega\}$ and define

$$
\gamma_{i k} \doteq \gamma=\exp \left[-\varepsilon^{\star}(\eta+\max \{\tau, \omega\})\right] \cdot \exp [-\mathbb{A} \eta]-\mathbb{B}-1
$$

As a special case of system (2.1), we consider the following neural network model:

$$
\dot{x}_{i}(t)=-d_{i} x_{i}(t)+\sum_{j=1}^{n} a_{i j} f_{j}\left(x_{j}(t)\right)+\sum_{j=1}^{n} b_{i j} g_{j}\left(\int_{0}^{\omega} K_{i j}(s) x_{j}(t-s) d s\right)+I_{i,} \quad t \geq t_{0}, i \in \Lambda .
$$

we can obtain theorem as follows.

Theorem 3.4. Assume that $\left(H_{1}\right)-\left(H_{3}\right)$ hold, then the equilibrium point of the system (3.19) can be exponentially stabilized by impulses if one of the following conditions holds

$\left(H_{4}\right) \mathbb{D}<0$.

$\left(H_{5}\right) \mathbb{E} \geq 0$ and $\exp [\mathbb{D} \eta] \cdot \mathbb{E}<1$, where

$$
\begin{aligned}
& \mathbb{D}=-\min _{i \in \Lambda} d_{i}+\sum_{i=1}^{n} \max _{j \in \Lambda}\left|a_{i j}\right| L_{j}^{f}+\sum_{i=1}^{n} \max _{j \in \Lambda}\left|b_{i j}\right| L_{j}^{g} \int_{0}^{\omega} \mathcal{K}(s) d s, \\
& \mathbb{E}=\sum_{i=1}^{n} \max _{j \in \Lambda}\left|b_{i j}\right| L_{j}^{g} \int_{0}^{\omega} \mathcal{K}(s) s d s .
\end{aligned}
$$

Proof. In fact, we need only to mention a few points since the rest is the same as in the proof of Theorem 3.1. First, instead of (3.4) we can get that

$$
D^{+} V(t) \leq \mathbb{D} V(t), \quad t \geq t_{0} .
$$


Second, instead of (3.6) and (3.7) we choose constants $\varepsilon^{\star}>0$ and $\eta \geq \omega$ such that

$$
\mathbb{E} \leq \exp \left[-\varepsilon^{\star}(\eta+\omega)\right] \exp [-\mathbb{D} \eta]
$$

Then one may choose a sequence $\left\{t_{k}\right\}_{k \in \mathbb{Z}_{+}}$such that $\omega \leq t_{k}-t_{k-1} \leq \eta$ and define

$$
\gamma_{i k}=\exp \left[-\varepsilon^{\star}\left(t_{k+1}-t_{k}+\omega\right)\right] \cdot \exp \left[-\mathbb{D}\left(t_{k+1}-t_{k}\right)\right]-\mathbb{E}-1 \text {. }
$$

Corollary 3.5. Assume that conditions in Theorem 3.4 hold, then the equilibrium point of the system (3.19) can be exponentially stabilized by periodic impulses.

Proof. Here we need only to choose the sequence $\left\{t_{k}\right\}_{k \in \mathbb{Z}_{+}}$such that $t_{k}-t_{k-1}=\eta \geq \omega$. Let

$$
\gamma_{i k} \doteq \gamma=\exp \left[-\varepsilon^{\star}(\eta+\omega)\right] \cdot \exp [-\mathbb{D} \eta]-\mathbb{E}-1 \text {. }
$$

\section{A Numerical Example}

In this section, we give an example to demonstrate the effectiveness of our method.

Example 4.1. Consider the following neural network consisting two neurons:

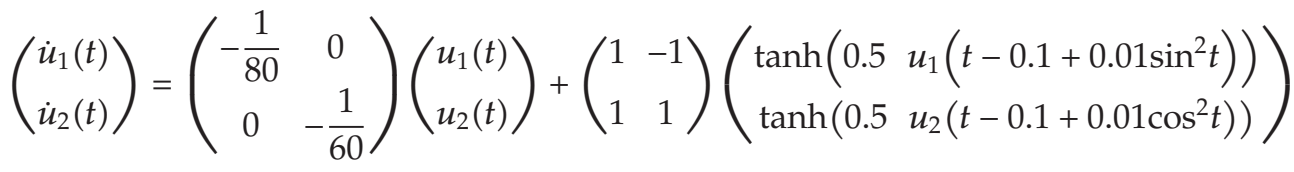

$$
\begin{aligned}
& +\left(\begin{array}{cc}
1 & 1 \\
-1 & 1
\end{array}\right)\left(\begin{array}{c}
\tanh \left(\int_{0}^{0.2} s u_{1}(t-s) d s\right) \\
\tanh \left(\int_{0}^{0.2} s u_{2}(t-s) d s\right)
\end{array}\right), \quad t \geq 0
\end{aligned}
$$

Then $L_{j}^{f}=0.5, L_{j}^{g}=1, j=1,2, \mathcal{K}(s)=s, \tau=0.1, \rho=0.01$, and $\omega=0.2$. It is obvious that $(0,0)^{T}$ is an equilibrium point of system (4.1). By simple calculation, we get

$$
\begin{gathered}
\mathbb{A}=-\min _{i \in \Lambda} d_{i}+\frac{1}{1-\rho} \sum_{i=1}^{n} \max _{j \in \Lambda}\left|a_{i j}\right| L_{j}^{f}+\sum_{i=1}^{n} \max _{j \in \Lambda}\left|b_{i j}\right| L_{j}^{g} \int_{0}^{\omega} \mathcal{K}(s) d s=1.0376>0, \\
\mathbb{B}=\frac{\tau}{1-\rho} \sum_{i=1}^{n} \max _{j \in \Lambda}\left|a_{i j}\right| L_{j}^{f}+\sum_{i=1}^{n} \max _{j \in \Lambda}\left|b_{i j}\right| L_{j}^{g} \int_{0}^{\omega} \mathcal{K}(s) s d s \approx 0.1410, \\
\exp [\operatorname{Amax}\{\tau, \omega\}] \cdot \mathbb{B} \approx 0.1735<1 .
\end{gathered}
$$

In this case, one may choose $\varepsilon^{\star}=0.01, t_{k}-t_{k-1}=0.2, \gamma_{1 k}=\gamma_{2 k}=-0.3316$ such that (3.6) and (3.7) in Theorem 3.1 hold. According to Theorem 3.1, the equilibrium point $[0,0]^{T}$ of system (4.1) can be exponentially stabilized by impulses. The numerical simulation is shown in Figures 1(b) and 1(e). 


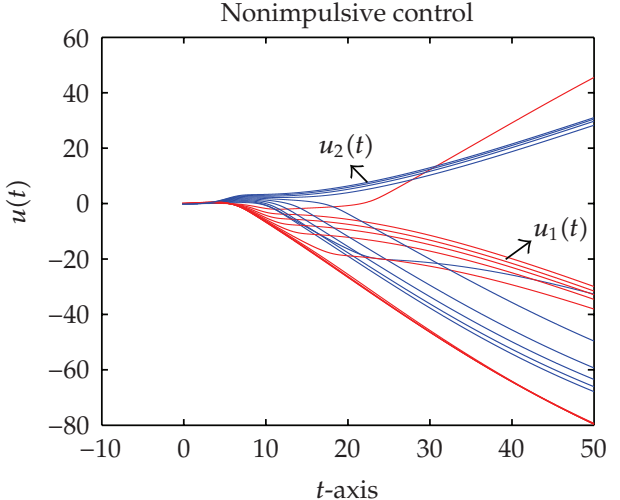

(a)

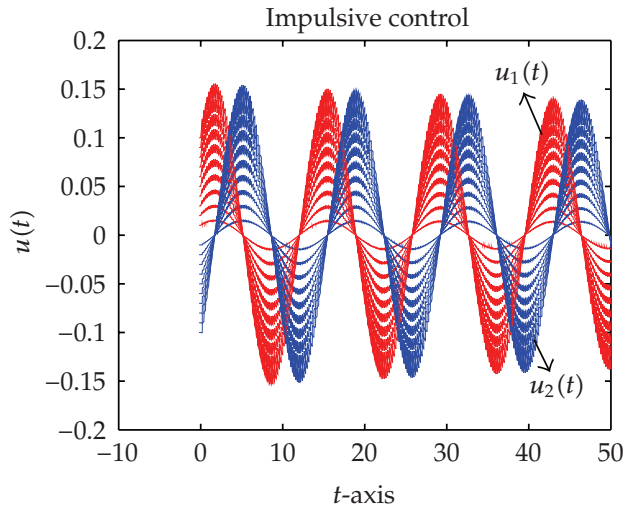

(c)

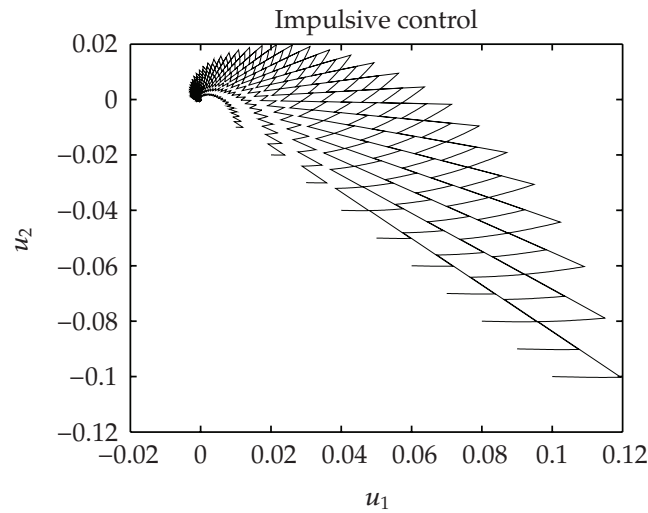

(e)

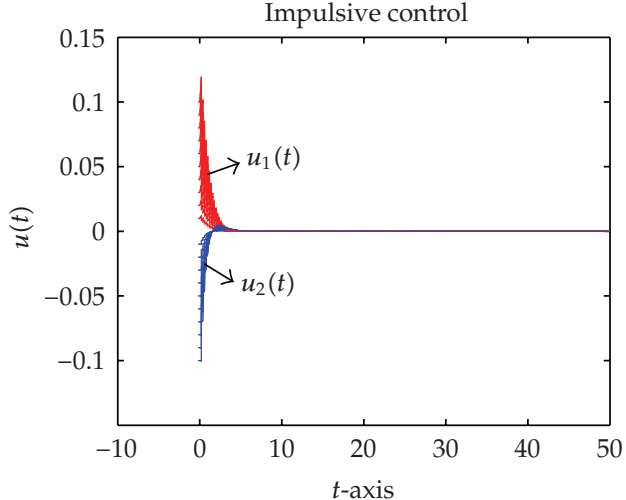

(b)

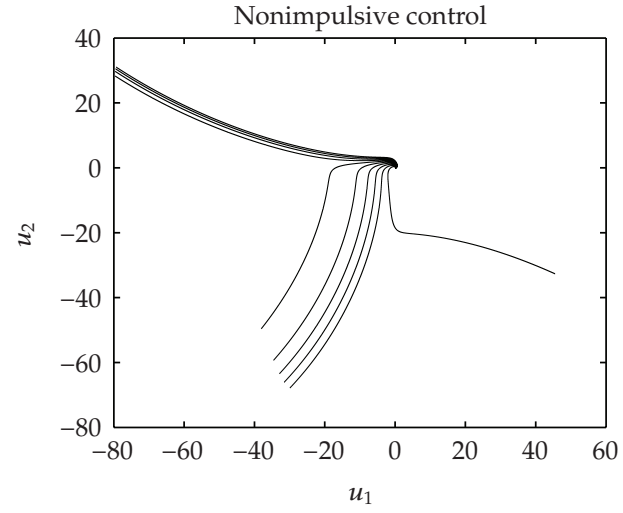

(d)

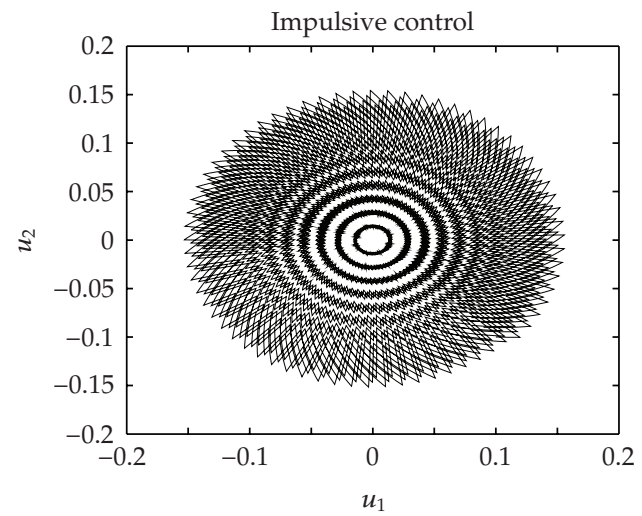

(f)

Figure 1: (a) Time-series of the $u$ of system (4.1) without impulsive control for $t \in[-0.2,50]$. (b) Time-series of the $u$ of system (4.1) by impulsive control with $\gamma_{1 k}=\gamma_{2 k}=-0.3316$ for $t \in[-0.2,50]$. (c) Time-series of the $u$ of system (4.1) by impulsive control with $\gamma_{1 k}=\gamma_{2 k}=-0.1$ for $t \in[-0.2,50]$. (d) Phase portrait of system (4.1) without impulsive control for $t \in[-0.2,50]$. (e) Phase portrait of system (4.1) by impulsive control with $\gamma_{1 k}=\gamma_{2 k}=-0.3316$ for $t \in[-0.2,50]$. (f) Phase portrait of system (4.1) by impulsive control with $\gamma_{1 k}=\gamma_{2 k}=-0.1$ for $t \in[-0.2,50]$. 
Remark 4.2. Note that $\gamma_{1 k}=\gamma_{2 k}=-0.3316$, by Corollary 3.3, system (4.1) can be exponentially stabilized by periodic impulses.

Remark 4.3. As we see from Figures 1 (a) and 1(d), the equilibrium point $[0,0]^{T}$ of system (4.1) without impulses is unstable. However, it becomes exponentially stable by explicit impulsive control (see Figures 1(b) and 1(e)). This implies that impulses may be used to exponentially stabilize some unable neural networks by our proposed control method. Furthermore, in the same impulse interval, if $\gamma_{1 k}=\gamma_{2 k}=-0.1$, then our control method in (3.6) and (3.7) is not satisfied. The equilibrium point $(0,0)^{T}$ of system $(4.1)$ cannot be exponentially stabilized by impulses, which is shown in Figures 1(c) and 1(f). However, one may observe that every solution of system (4.1) becomes a quasiperiodic solution because of the effects of impulses. Figures $1(\mathrm{a})-1(\mathrm{f})$ show the dynamic behavior of the system (4.1) with the initial condition $\left[u_{1}(t), u_{2}(t)\right]^{T}=[s N,-s N]^{T}, N=1,2, \ldots, 10, s=0.01, t \in[-0.2,0]$.

\section{Conclusions}

In this paper, we have investigated impulsive control for neural networks with both time-varying and distributed delays. By using Lyapunov functionals, stability theory, and control by impulses, some sufficient conditions are derived to exponentially stabilize neural networks with both time-varying and distributed delays. Simulation results of a neural network under impulsive control verify the effectiveness of the proposed control method.

\section{Acknowledgment}

The work is supported by the Science and Technology Programs of Shandong Province (2008GG30009008).

\section{References}

[1] J. H. Park, C. H. Park, O. M. Kwon, and S. M. Lee, "A new stability criterion for bidirectional associative memory neural networks of neutral-type," Applied Mathematics and Computation, vol. 199, no. 2, pp. 716-722, 2008.

[2] J. Cao, J. Liang, and J. Lam, "Exponential stability of high-order bidirectional associative memory neural networks with time delays," Physica D, vol. 199, no. 3-4, pp. 425-436, 2004.

[3] K. Gopalsamy, "Stability of artificial neural networks with impulses," Applied Mathematics and Computation, vol. 154, no. 3, pp. 783-813, 2004.

[4] J. Hopfield, "Neurons with graded response have collective computational properties like those of two-state neurons," Proceedings of the National Academy of Sciences of the United States of America, vol. 81, no. 10, pp. 3088-3092, 1984.

[5] M. Liu, "Global asymptotic stability analysis of discrete-time Cohen-Grossberg neural networks based on interval systems," Nonlinear Analysis: Theory, Methods \& Applications, vol. 69, no. 8, pp. 24032411, 2008.

[6] Z. Huang and Y. Xia, "Global exponential stability of BAM neural networks with transmission delays and nonlinear impulses," Chaos, Solitons E Fractals, vol. 38, no. 2, pp. 489-498, 2008.

[7] J. Zhang, Z. Jin, J. Yan, and G. Sun, "Stability and Hopf bifurcation in a delayed competition system," Nonlinear Analysis: Theory, Methods E Applications, vol. 70, no. 2, pp. 658-670, 2009.

[8] X.-Y. Lou and B.-T. Cui, "Novel global stability criteria for high-order Hopfield-type neural networks with time-varying delays," Journal of Mathematical Analysis and Applications, vol. 330, no. 1, pp. 144 $158,2007$. 
[9] Z. Yang and D. Xu, "Global exponential stability of Hopfield neural networks with variable delays and impulsive effects," Applied Mathematics and Mechanics, vol. 27, no. 11, pp. 1517-1522, 2006.

[10] Y. Zhang and J. Sun, "Stability of impulsive neural networks with time delays," Physics Letters A, vol. 348, no. 1-2, pp. 44-50, 2005.

[11] M. Syed Ali and P. Balasubramaniam, "Robust stability for uncertain stochastic fuzzy BAM neural networks with time-varying delays," Physics Letters A, vol. 372, no. 31, pp. 5159-5166, 2008.

[12] X. Li and Z. Chen, "Stability properties for Hopfield neural networks with delays and impulsive perturbations," Nonlinear Analysis: Theory, Methods \& Applications, vol. 10, pp. 3253-3265, 2009.

[13] D. Xu and Z. Yang, "Impulsive delay differential inequality and stability of neural networks," Journal of Mathematical Analysis and Applications, vol. 305, no. 1, pp. 107-120, 2005.

[14] D. D. Bănnov and P. S. Simeonov, Systems with Impulse Effect: Stability, Theory and Applications, Ellis Horwood Series: Mathematics and Its Applications, Ellis Horwood, Chichester, UK, 1989.

[15] X. Fu, B. Yan, and Y. Liu, Introduction of Impulsive Differential Systems, Science Press, Beijing, China, 2005.

[16] X. Fu and X. Li, "W-stability theorems of nonlinear impulsive functional differential systems," Journal of Computational and Applied Mathematics, vol. 221, no. 1, pp. 33-46, 2008.

[17] X. Li, "Oscillation properties of higher order impulsive delay differential equations," International Journal of Difference Equations, vol. 2, no. 2, pp. 209-219, 2007.

[18] X. Fu and X. Li, "Oscillation of higher order impulsive differential equations of mixed type with constant argument at fixed time," Mathematical and Computer Modelling, vol. 48, no. 5-6, pp. 776-786, 2008.

[19] X. Liu, "Stability results for impulsive differential systems with applications to population growth models," Dynamics and Stability of Systems, vol. 9, no. 2, pp. 163-174, 1994.

[20] G. Ballinger and X. Liu, "Practical stability of impulsive delay differential equations and applications to control problems," in Optimization Methods and Applications, vol. 52 of Applied Optimization, pp. 3-21, Kluwer Academic Publishers, Dordrecht, The Netherlands, 2001.

[21] I. M. Stamova and G. T. Stamov, "Lyapunov-Razumikhin method for impulsive functional differential equations and applications to the population dynamics," Journal of Computational and Applied Mathematics, vol. 130, no. 1-2, pp. 163-171, 2001.

[22] J. Sun and Y. Zhang, "Impulsive control of Rössler systems," Physics Letters A, vol. 306, no. 5-6, pp. 306-312, 2003.

[23] Y. Li, X. Liao, C. Li, T. Huang, and D. Yang, "Impulsive synchronization and parameter mismatch of the three-variable autocatalator model," Physics Letters A, vol. 366, no. 1-2, pp. 52-60, 2007.

[24] Y. Zhang and J. Sun, "Controlling chaotic Lu systems using impulsive control," Physics Letters A, vol. 342 , no. 3, pp. 256-262, 2005.

[25] B. Liu, K. L. Teo, and X. Liu, "Robust exponential stabilization for large-scale uncertain impulsive systems with coupling time-delays," Nonlinear Analysis: Theory, Methods E Applications, vol. 68, no. 5, pp. 1169-1183, 2008.

[26] Z. Yang and D. Xu, "Impulsive effects on stability of Cohen-Grossberg neural networks with variable delays," Applied Mathematics and Computation, vol. 177, no. 1, pp. 63-78, 2006.

[27] D. Li, S. Wang, X. Zhang, and D. Yang, "Impulsive control of uncertain Lotka-Volterra predator-prey system," Chaos, Solitons E Fractals. In press.

[28] Q. Wang and X. Liu, "Impulsive stabilization of delay differential systems via the LyapunovRazumikhin method," Applied Mathematics Letters, vol. 20, no. 8, pp. 839-845, 2007.

[29] A. Weng and J. Sun, "Impulsive stabilization of second-order delay differential equations," Nonlinear Analysis: Real World Applications, vol. 8, no. 5, pp. 1410-1420, 2007.

[30] Z. Luo and J. Shen, "Impulsive stabilization of functional differential equations with infinite delays," Applied Mathematics Letters, vol. 16, no. 5, pp. 695-701, 2003.

[31] Z. Luo and J. Shen, "New Razumikhin type theorems for impulsive functional differential equations," Applied Mathematics and Computation, vol. 125, no. 2-3, pp. 375-386, 2002.

[32] X. Liu, "Impulsive stabilization of nonlinear systems," IMA Journal of Mathematical Control and Information, vol. 10, no. 1, pp. 11-19, 1993. 